Cell biology

\title{
Sight at the end of the tunnel
}

Arthur Horwich

A chaperone molecule called trigger factor binds new polypeptide chains as they emerge from the protein-synthesis machinery. Crystal structures suggest that this molecule forms a hydrophobic 'cradle'.

C ells seem to leave nothing to chance, including the final step of information transfer - the folding of a newly made chain of amino acids into a threedimensional, active, 'native' protein. Specialized proteins called molecular chaperones ensure that the process of folding, determined by the amino-acid sequence of a polypeptide chain, does not go awry ${ }^{1,2}$. On page 590 of this issue, Ferbitz et al. ${ }^{3}$ present crystallographic images of a bacterial chaperone called trigger factor. The images provide clues to how this molecule interacts with the newly synthesized polypeptide chain as it emerges from a tunnel in the protein-synthesizing machinery (the ribosome), potentially cradling and protecting segments of the polypeptide.

Chaperones typically assist the folding process by specifically binding to polypeptides through a feature that is unique to non-native proteins - exposed hydrophobic surfaces. These surfaces become buried in the interior of a protein in its final form. Such hydrophobic regions, left to their own devices, can bind to each other, producing aggregates, which are not only

Konstanzer Online-Publikations-System (KOPS) URL: http://www.ub.uni-konstanz.de/kops/volltexte/2007/3733/ URN: http://nbn-resolving.de/urn:nbn:de:bsz:352-opus-37339 a dead-end for protein function but also potentially toxic to the cell; for example, aggregates are found in several neurodegenerative diseases. Chaperones intervene by binding these exposed surfaces through a hydrophobic site of their own, preventing aggregation and enabling productive folding when the chaperoned protein is released.

The long-awaited structure of the triggerfactor chaperone, presented by Ferbitz et al. ${ }^{3}$, reveals an extended arrangement of three domains - a 'crouching dragon' with a head, tail and arms - and a notable hydrophobic surface in the shape of a cradle that is exposed in the hollow between the tail and arms. Excitingly, Ferbitz et al. place this in a functional context by means of a second structure. This structure shows the tail portion of trigger factor in complex with the large subunit of the ribosome, suggesting the position of intact trigger factor as it might interact with the ribosome.

This second structure is a considerable technical achievement, involving astute evolutionary considerations, incisive biochemical analysis and some deft crystallography. The only ribosomal large subunit that has been observed at high resolution by X-ray crystallography is that from the archaeon Haloarcula marismortui. The structure of this subunit, presented several years ago ${ }^{4}$, provided unprecedented resolution of such features as the reaction centre, where peptide bonds are formed, and the exit tunnel. But archaea lack trigger factor, instead using other molecules to protect nascent chains.

The investigators had previously identified $^{5}$ the contact site for trigger factor on the ribosome of the bacterium Escherichia coli, 

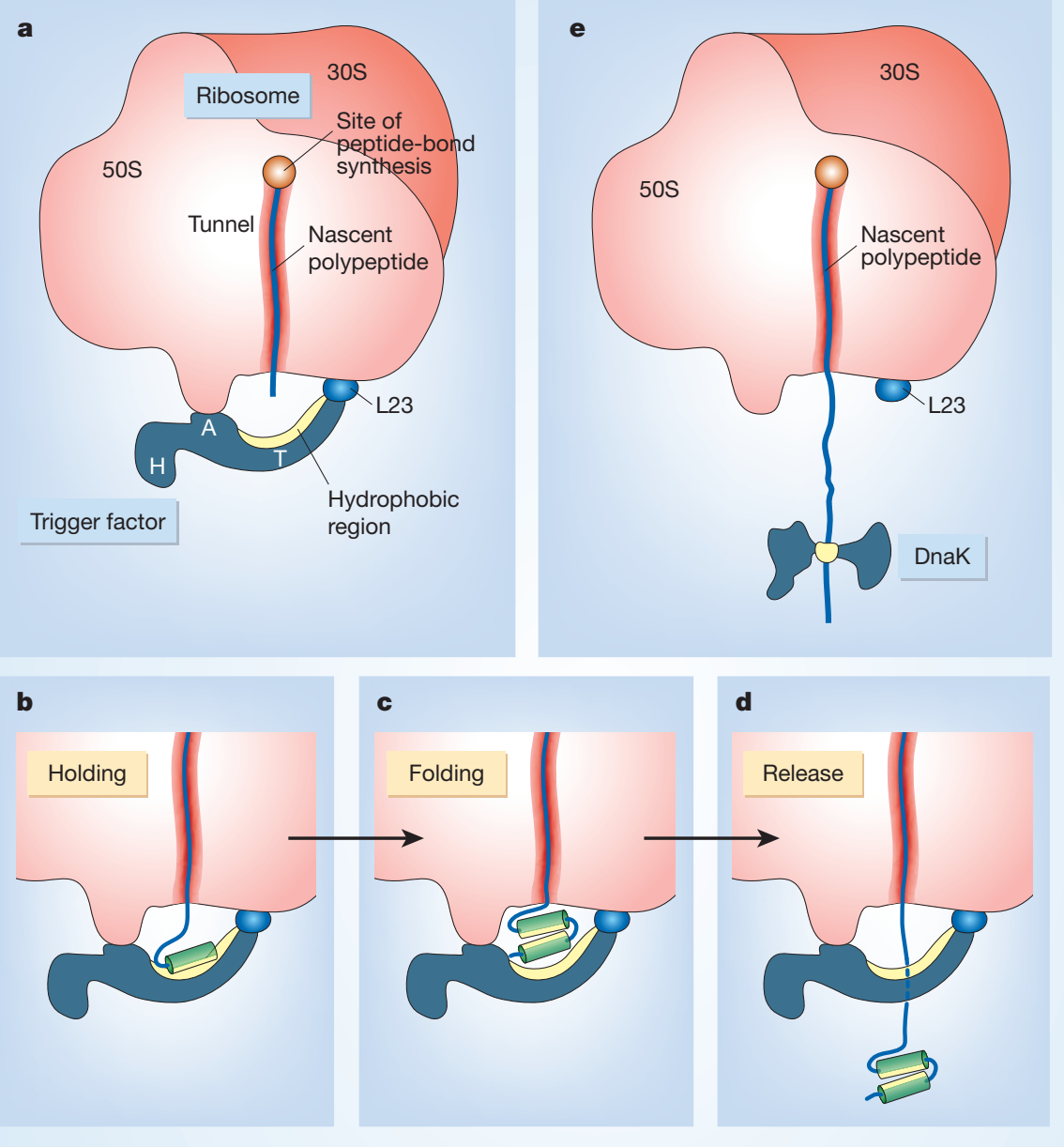

Figure 1 Chaperones at the ribosome. a, Ferbitz et al. ${ }^{3}$ have determined the crystal structure of the trigger-factor tail domain $(\mathrm{T})$ in complex with a ribosome large subunit $(50 \mathrm{~S})$, and used this to position their crystallographic model of intact trigger factor, including the $\operatorname{arm}(\mathrm{A})$ and head $(\mathrm{H})$ domains. Trigger factor forms a cavity, with a hydrophobic lining, at the exit of a tunnel in the ribosome from which newly made polypeptide chains emerge. Its main contacts are with the ribosomal protein L23. b-d, How the nascent polypeptide might behave. b, 'Holding', where hydrophobic sections (yellow) of the nascent structure (here on an $\alpha$-helix, symbolized by a barrel) could be stabilized by binding to trigger factor's hydrophobic surface. c, 'Folding', where a further stretch of emerging chain docks with that of the first, forming a substructure within the cavity. d, 'Release', where the substructure dissociates from trigger factor, by an unknown mechanism, and a new segment of chain enters the cavity. $e$, The binding of nascent polypeptide chains by chaperone DnaK is entirely different, and involves a narrow hydrophobic arch in DnaK ${ }^{9}$ (only the peptide-binding domain of DnaK is shown). The nascent chain might also acquire $\alpha$-helical structure within the ribosomal tunnel ${ }^{12,13}$, but this is not shown here.

finding that ribosomal protein L23 is particularly important. They reasoned that, because this contact site is highly conserved across the evolutionary kingdoms, a crosskingdom complex of archaeal ribosome subunit and bacterial trigger factor should be possible. And they were right. Remarkably, the ribosome-binding tail domain of trigger factor, comprising 144 amino acids (out of 432), can bind to and crystallize as a complex with the ribosomal subunit. This allowed Ferbitz and colleagues to resolve the structure of part of this domain, consisting of the 40 amino acids that lie nearest to the ribosomal subunit.

Gratifyingly, the contacts that trigger factor makes with the large subunit are right next to the exit of the ribosomal tunnel, and are made principally with the L23 protein. The authors then used the architecture of this fragment of trigger factor to superpose the entire stand-alone trigger-factor structure in relation to the ribosome. Startlingly, the chaperone seems to be positioned over the ribosomal tunnel exit, with its hydrophobic cradle providing a cavity into which the nascent chain would emerge (Fig. 1a).

The idea is inescapable that this cavity could produce a unique environment that can both physically protect the emerging nascent chain from protease enzymes (which could readily cleave a weakly structured polypeptide chain) and allow the stabilization of emerging hydrophobic parts of the nascent chain (Fig. 1b). Furthermore, the cavity is large enough $(30 \times 35 \times \sim 20 \AA)$ to allow segments of the emerging nascent chain to fold partially inside it (Fig. 1c). For example, a local region, such as a protein domain, could potentially fold into its native form inside this space. Indeed, Ferbitz et al. used computer docking to show that the native form of the small protein lysozyme could fit into the space.

This model is very appealing, but it also raises a number of questions, not least about whether the positioning of the entire standalone model of trigger factor in relation to the ribosome is valid. For instance, might there be a structural rearrangement as the trigger factor binds to the ribosomenascent-chain complex? There is a striking precedent for such a rearrangement: the so-called signal-recognition particle also associates with the ribosome L23 region, and it bends hugely when it binds to a ribosomenascent-chain complex ${ }^{6}$. Such a transition would not necessarily be expected for trigger factor on functional grounds, but electron micrographs of ribosome-bound trigger factor with and without a nascent chain present would address this.

How general might the proposed mechanism for trigger factor be? A chaperone known as DnaK can entirely replace trigger factor in supporting protein folding ${ }^{7,8}$. But, rather than forming a hydrophobic cradle, DnaK instead binds hydrophobic stretches of nascent chains through a small hydrophobic archway of its own ${ }^{9}$ (Fig. 1e). This suggests that a trigger-factor-like cavity is not essential to sustain correct protein folding.

It also remains unclear how the elongating polypeptide leaves trigger factor (Fig. 1d). Kinetic studies ${ }^{10}$ have suggested that trigger factor binds to the ribosome long enough for an entire chain to be synthesized, although these studies were done in the absence of polypeptide. This would imply either that the nascent chain exits through a side passage (front or back in Fig. 1) or that trigger factor changes its conformation to allow the nascent chain out. Alternatively, the growing nascent polypeptide might itself affect the residence time of trigger factor at the ribosome. Although several studies have suggested that nascent chains stabilize complexes of trigger factor with ribosomes (see, for example, ref. 11), perhaps transient release of trigger factor from the ribosome does nonetheless occur, such that the polypeptide controls the opening of the cavity and its own release.

More general questions about chaperone mechanisms also remain. Why haven't chaperones that interact with nascent chains been highly conserved in evolution (trigger factor, for instance, is found only in bacteria)? Are there kingdom-specific requirements that are related to particular proteomes or to cellular growth conditions? Or are there simply 


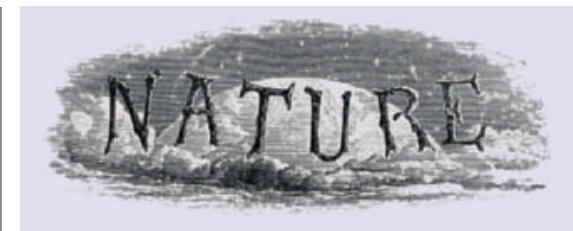

100 YEARS AGO

The inability of a large number of skilful experimental physicists to obtain any evidence whatever of the existence of the $n$-rays, and the continued publication of papers announcing new and still more remarkable properties of the rays, prompted me to pay a visit to one of the laboratories in which the apparently peculiar conditions necessary for the manifestation of this most elusive form of radiation appear to exist. I went, I must confess, in a doubting frame of mind, but with the hope that I might be convinced of the reality of the phenomena, the accounts of which have been read with so much scepticism... I am obliged to confess that I left the laboratory with a distinct feeling of depression, not only having failed to see a single experiment of a convincing nature, but with the almost certain conviction that all the changes in the luminosity or distinctness of sparks and phosphorescent screens (which furnish the only evidence of $n$-rays) are purely imaginary. It seems strange that after a year's work on the subject not a single experiment has been devised which can in any way convince a critical observer that the rays exist at all.

R. W. Wood From Nature 29 September 1904.

\section{YEARS AGO}

Jean Piaget's reputation as a psychologist in Great Britain is largely based upon a series of books written during 1925-32 dealing with the development of thought, language and moral judgment in the child. But, as he himself points out, this work was merely a prolegomena to his later investigations extending from 1937 to the present day... But though these researches are both theoretically and experimentally an advance upon his earlier work, they have, however, had little effect on English psychological thought... This is probably due to Piaget's introduction of a new and complex terminology, his use of symbolic logic, and the fact that his most important work remains untranslated... The most interesting conclusion which emerges from this important series of experimental researches is that mathematical concepts in their psychological development are ultimately based upon simple logical notions. Indeed, it might be said, without undue exaggeration, that Piaget's psychological studies are the genetic counterpart of Russell and Whitehead's attempt in "Principia Mathematica" to put mathematics on to a logical basis.

From Nature 2 October 1954. many ways of bringing about a low-affinity, temporary interaction with a nascent chain - many ways for a chaperone 'midwife' to hold the baby?

Arthur Horwich is in the Department of Genetics and the Howard Hughes Medical Institute, Boyer

Center, Yale School of Medicine, New Haven,

Connecticut 06510, USA, and The Scripps Research

Institute, La Jolla, California, USA.

1. Bukau, B., Deuerling, E., Pfund, C. \& Craig, E. A. Cell 101, 119-122 (2000).

2. Hartl, F. U. \& Hayer-Hartl, M. Science 295, 1852-1858 (2002).

3. Ferbitz, L. et al. Nature 431, 590-596 (2004).
4. Ban, N., Nissen, P., Hansen, J., Moore, P. B. \& Steitz, T. A. Science 289, 905-920 (2000).

5. Kramer, G. et al. Nature 419, 171-174 (2002).

6. Halic, M. et al. Nature 427, 808-814 (2004).

7. Deuerling, E., Schulze-Specking, A., Tomoyasu, T., Mogk, A. \& Bukau, B. Nature 400, 693-696 (1999).

8. Teter, S. A. et al. Cell 97, 755-765 (1999).

9. Zhu, X. et al. Science 272, 1606-1614 (1996).

10. Maier, R., Eckert, B., Scholz, C., Lilie, H. \& Schmid, F. -X. J. Mol. Biol. 326, 585-592 (2003).

11. Hesterkamp, T., Hauser, S., Lütcke, H. \& Bukau, B. Proc. Natl Acad. Sci. USA 93, 4437-4441 (1996).

12. Mingarro, I. et al. BMC Cell Biol. 1, 3 (2000).

13. Woolhead, C. A., McCormick, P. J. \& Johnson, A. E. Cell 116, 725-736 (2004). 